\title{
POLARIZATION EFFECTS IN NON-LINEAR SCATTERING
}

\author{
D.L. ANDREWS and T. THIRUNAMACHANDRAN \\ Department of Chemistry, University College, London WCI, England
}

Received 24 June 1977

\begin{abstract}
The problem of non-linear scattering of unpolarized light is discussed. New relationships between the scattering intensities for unpolarized and polarized incident light are given. It is shown that the usual equation connecting the depolarization ratios for plane and unpolarized light does not hold for non-linear scattering processes such as hyper-Rayleigh and hyperRaman scattering. A new relation between the two is given in terms of two additional intensity ratios. An expression for the depolarization ratio for unpolarized light is also given in terms of the hyperpolarizability tensor.
\end{abstract}

In Rayleigh and Raman scattering studies, an important measurable quantity is the depolarization ratio or degree of depolarization. For such processes, the depolarization ratios for plane polarized and unpolarized incident light provide the same information about the scattering system, and a well-known simple relation exists between them. In the study [1-3] of higher order processes such as elastic second-harmonic light scattering, known as hyper-Rayleigh scattering, and its inelastic analogue, hyper-Raman scattering, the same relation has been assumed to hold. Here we show that this simple relation is inapplicable for these and other higher order processes, and that with two new intensity ratios it is possible to relate the polarization ratios for polarized and unpolarized light.

The polarization ratios which we are concerned with are defined as the ratio of intensities

$$
\rho_{\lambda}(\theta)=\left[I_{\theta}(\lambda \rightarrow \|)\right] /\left[I_{\theta}(\lambda \rightarrow 1)\right]
$$

where $\theta$ is the scattering angle; $\|$ and $\perp$ denote the polarization of scattered light resolved into orthogonal components lying respectively in and normal to the scat tering plane, i.e. the plane containing the incident and emergent directions of light propagation. Also, $\lambda$ stands for $\perp$ or $u$; the former refers to the incident photon with polarization perpendicular to the scattering plane, and the latter to unpolarized incident light, i.e. a statistical mixture of equal weights of two orthogonal polarizations. In general, the intensity of Rayleigh and Raman scattering of unpolarized light is given by

$$
I_{\theta}(\mathrm{u} \rightarrow \mu)=\frac{1}{2}\left[I_{\theta}(\| \rightarrow \mu)+I_{\theta}(\perp \rightarrow \mu)\right]=\frac{1}{2}\left[I_{\theta}(\mathrm{L} \rightarrow \mu)+I_{\theta}(\mathrm{R} \rightarrow \mu)\right],
$$

where $\mu$ refers to either plane or circular $(\mathrm{L} / \mathrm{R})$ polarization of the scattered photon. With the aid of (2) and Krishnan's relations [4],

$$
I_{\theta}(\| \rightarrow \perp)=I_{\theta}(\perp \rightarrow \|)=I_{\pi / 2}(\|\rightarrow\|) \text {, }
$$

we obtain the usual relationship between the depolarization ratios,

$$
\rho_{u}(\pi / 2)=2 \rho_{\perp} /\left(1+\rho_{\perp}\right)
$$

we note that $\rho_{\perp}$ is independent of the scattering angle.

For non-linear scattering, eq. (4) relating $\rho_{\perp}$ to $\rho_{u}(\pi / 2)$ does not hold [5]. Although Krishnan's relations (3) remain valid, eq. (2) relating the intensity of scattering of unpolarized light with that of polarized light is inapplicable to elastic or inelastic harmonic scattering. It is easily shown that since the polarizations of the incident photons of an unpolarized beam are not correlated, the analogue of (2) for $n$-harmonic scattering is 


$$
I_{\theta}(\mathrm{u} \rightarrow \mu)=\frac{1}{2^{n}} \sum_{m=0}^{n}{ }^{n} C_{m} I_{\theta}[(n-m) \|, m \perp \rightarrow \mu],
$$

where $I_{\theta}[(n-m) \|, m \perp \rightarrow \mu]$ refers to the absorption of $m$ photons with perpendicular polarization and $(n-m)$ with parallel polarization, together with the emission of a single frequency-multiplied photon of polarization $\mu$. In particular, for hyper-Rayleigh or hyper-Raman scattering we have

$$
I_{\theta}(\mathrm{u} \rightarrow \mu)=\frac{1}{4}\left[I_{\theta}(2 \| \rightarrow \mu)+2 I_{\theta}(\|, \perp \rightarrow \mu)+I_{\theta}(2 \perp \rightarrow \mu)\right] .
$$

It is the omission of the term $2 I_{\theta}(\|, \perp \rightarrow \mu)$ that leads to the inapplicability of eq. (4) for these three photon processes. In single-beam experiments, this particular term is not directly measurable. However, it can be shown that, within the electric dipole approximation, an equivalent form of (6) in terms of directly measurable quantities is

$$
I_{\theta}(\mathrm{u} \rightarrow \mu)=\frac{1}{8}\left[2 I_{\theta}(2 \| \rightarrow \mu)+2 I_{\theta}(2 \perp \rightarrow \mu)+I_{\theta}(2 \mathrm{~L} \rightarrow \mu)+I_{\theta}(2 \mathrm{R} \rightarrow \mu)\right] .
$$

It is of interest to note that the analogues of (6) and (7) for the rates of two-photon absorption of unpolarized light are

$$
\Gamma(\mathrm{u})=\frac{1}{2}[\Gamma(2 \|)+\Gamma(\|, \perp)] \equiv \frac{1}{4}[2 \Gamma(2 \|)+\Gamma(2 \mathrm{c})],
$$

with obvious equality $\Gamma(2 \|)=\Gamma(2 \perp)$; denotes circular polarization of either handedness.

Returning to hyper-Rayleigh and hyper-Raman scattering, we now make use of eq. (7) to write down a new expression which correctly relates $\rho_{u}(\pi / 2)$ and $\rho_{\perp}$. For this purpose, we introduce two new parameters $\sigma_{\|}$and $\sigma_{\perp}$ which measure the relative intensities of scattering of circularly polarized and plane polarized light. They are defined by

$$
\sigma_{\mu}(\theta)=\left[I_{\theta}(2 \mathrm{c} \rightarrow \mu)\right] /\left[I_{\theta}(2 \perp \rightarrow \mu)\right] \quad(\mu=\|, \perp) .
$$

We note that $\sigma_{\| i}$, unlike $\sigma_{\perp}$, is a $\theta$-dependent quantity. It is now readily shown that $\rho_{u}(\pi / 2)$ is given in terms of $\rho_{\perp}$, $\sigma_{\|}(\pi / 2)$ and $\sigma_{\perp}$ by

$$
\rho_{u}(\pi / 2)=\rho_{\perp}\left(2+\sigma_{\|}(\pi / 2)\right) /\left(1+\rho_{\perp}+\sigma_{\perp}\right) .
$$

The general expression for $\rho_{u}(\theta)$ takes the same form as for Rayleigh or Raman scattering, namely,

$$
\rho_{u}(\theta)=\rho_{u}(\pi / 2)+\left[1-\rho_{u}(\pi / 2)\right] \cos ^{2} \theta
$$

It is possible to write down explicit expressions for $\rho_{u}(\pi / 2)$ in terms of the hyperpolarizability tensor $\beta_{\lambda \mu \nu}$. The result for hyper-Rayleigh scattering is

$$
\rho_{u}(\pi / 2)=\frac{4 \beta_{\lambda \lambda \mu} \beta_{\mu \nu \nu}-10 \beta_{\lambda \lambda \mu} \beta_{\nu \nu \mu}+\beta_{\lambda \mu \mu} \beta_{\lambda \nu \nu}+23 \beta_{\lambda \mu \nu} \beta_{\lambda \mu \nu}-10 \beta_{\lambda \mu \nu} \beta_{\mu \lambda \nu}}{-2 \beta_{\lambda \lambda \mu} \beta_{\mu \nu \nu}+5 \beta_{\lambda \lambda \mu} \beta_{\nu \nu \mu}+3 \beta_{\lambda \mu \mu} \beta_{\lambda \nu \nu}+13 \beta_{\lambda \mu \nu} \beta_{\lambda \mu \nu}+5 \beta_{\lambda \mu \nu} \beta_{\mu \lambda \nu}},
$$

where

$$
\begin{aligned}
\beta_{\lambda \mu \nu} & =\frac{1}{2} \sum_{r, s}\left[\frac{\mu_{\lambda}^{0 s} \mu_{\mu}^{s r} \mu_{\nu}^{r 0}}{\left(E_{s 0}-2 \hbar c k\right)\left(E_{r 0}-\hbar c k\right)}+\frac{\mu_{\mu}^{0 s} \mu_{\lambda}^{s r} \mu_{\nu}^{r 0}}{\left(E_{s 0}+\hbar c k\right)\left(E_{r 0}-\hbar c k\right)}+\frac{\mu_{\mu}^{0 s} \mu_{\nu}^{s r} \mu_{\lambda}^{r 0}}{\left(E_{s 0}+\hbar c k\right)\left(E_{r 0}+2 \hbar c k\right)}\right. \\
+ & \left.\frac{\mu_{\lambda}^{0 s} \mu_{\nu}^{s r} \mu_{\mu}^{r 0}}{\left(F_{s 0}-2 \hbar c k\right)\left(E_{r 0}-\hbar c k\right)}+\frac{\mu_{\nu}^{0 s} \mu_{\lambda}^{s r} \mu_{\mu}^{r 0}}{\left(E_{s 0}+\hbar c k\right)\left(E_{r 0}-\hbar c k\right)}+\frac{\mu_{\nu}^{0 s} \mu_{\mu}^{s r} \mu_{\lambda}^{r 0}}{\left(E_{s 0}+\hbar c k\right)\left(E_{r 0}+2 \hbar c k\right)}\right] .
\end{aligned}
$$

The result for hyper-Raman scattering follows from (13) by replacement of $\beta_{\lambda \mu \nu}$ by $\beta_{\lambda \mu \nu}^{\prime}$, the derivative with respect to a given vibrational mode coordinate. The hyperpolarizability tensor $\beta_{\lambda \mu \nu}$ has only $\mu, \nu$ index symmetry; these indices are associated with the two incident photons which are absorbed in the non-linear scattering process. In early work [1], the $\beta$-tensor was assumed to be symmetric in all pairs of indices; as was pointed out by Christie and Lockwood [6], this assumption is valid only in the limit of low incident frequencies. In this limit, (13) reduces to the simpler form, 


$$
\rho_{u}(\pi / 2)=\left(-5 \beta_{\lambda \mu \mu} \beta_{\lambda \nu \nu}+13 \beta_{\lambda \mu \nu} \beta_{\lambda \mu \nu}\right) /\left(6 \beta_{\lambda \mu \mu} \beta_{\lambda \nu \nu}+18 \beta_{\lambda \mu \nu} \beta_{\lambda \mu \nu}\right)
$$

which may be contrasted with expression (16), an equivalent form of the relation given in ref. [1]:

$$
\rho_{u}(\pi / 2)=\left(-\beta_{\lambda \mu \mu} \beta_{\lambda \nu \nu}+4 \beta_{\lambda \mu \nu} \beta_{\lambda \mu \nu}\right) /\left(4 \beta_{\lambda \mu \mu} \beta_{\lambda \nu \nu}+5 \beta_{\lambda \mu \nu} \beta_{\lambda \mu \nu}\right)
$$

The difference between (15) and (16) is due to the erroneous assumption of (4) in the calculation of (16). Full details of the theory and other aspects of hyper-Raman scattering will be described elsewhere.

We conclude by noting that for Rayleigh or Raman scattering, the obvious analogues of $\sigma_{\|}$and $\sigma_{\perp}$ are given by

$$
\sigma_{\|}(\pi / 2)=\left[I_{\pi / 2}(\mathrm{c} \rightarrow \|) /\left[I_{\pi / 2}(\perp \rightarrow \|)\right]=1, \quad \sigma_{\perp}=[I(\mathrm{c} \rightarrow \perp)] /[I(\perp \rightarrow \perp)]=\frac{1}{2}\left(1+\rho_{\perp}\right),\right.
$$

so that in this case (11) reduces to the standard result (4).

\section{References}

[1] S.J. Cyvin, J.E. Rauch and J.C. Recius, J. Chem. Phys. 43 (1965) 4083.

[2] S. Kielich and M. Kozierowski, Opt. Comm. 4 (1972) 395.

[3] S. Kielich, J.R. Lalane and F.B. Martin, J. Raman Spec. 1 (1973) 119.

[4] R.S. Krishnan, Proc. Ind. Acad. Sci. A1 (1935) 782.

[5] V.L. Strizhevskii and V.M. Klimenko, Sov. Phys. JETP 26 (1968) 163.

[6] J.H. Christie and D.J. Lockwood, J. Chem. Phys. 54 (1971) 1141. 